LA FORMACIÓN PROFESIONAL EN LA UNIVERSIDAD: UNA PROPUESTA A TRAVÉS DE LA EVALUACIÓN MEDIANTE CASOS AUTENTICOS. Páginas 271-286 en Revista de la Escuela de Ciencias de la Educación, año 10, número 9, enero a diciembre de 2014. ISSN 1851-6297. ISSN EN LINEA 2362-3349.

\title{
LA FORMACIÓN PROFESIONAL EN LA UNIVERSIDAD: UNA PROPUESTA A TRAVÉS DE LA EVALUACIÓN MEDIANTE CASOS AUTENTICOS*
}

\author{
Por María Florencia Di Matteo Demirdjian \\ (Universidad de Buenos Aires)*, Argentina. \\ florenciadm@hotmail.com
}

Recibido: 24/06/2014 Aceptado: 28/08/2014

\section{Resumen}

En este artículo presentamos las perspectivas de la docente y los estudiantes de un curso de la Licenciatura en Administración de la Universidad de Buenos Aires sobre la evaluación basada en casos auténticos y describimos las percepciones de los alumnos sobre sus modalidades de estudio para este tipo de evaluación. El estudio de caso se llevó a cabo desde una perspectiva cualitativa.

La evaluación de los aprendizajes a través de casos auténticos permite desarrollar habilidades requeridas para la formación profesional. Las demandas de uso aplicado de conocimientos y de elaboración de estrategias de acción sobre los casos reales posicionan al estudiante como futuro profesional en un marco institucional cuyas prácticas de enseñanza predominantes son de desarrollos conceptuales. La modalidad de evaluación se acompaña por una propuesta de enseñanza que implica el trabajo en equipo, el desarrollo de la oralidad y la autoevaluación. La noción de autenticidad se vincula con el carácter real de los casos. En relación con las modalidades de estudio los alumnos señalan una forma continua dada a partir de la resolución de tareas en clases y centrada en la adquisición de contenidos a partir de su aplicación en el análisis de los casos.

\section{Palabras Clave}

Evaluación de los aprendizajes - Método de casos - Formación profesional Metodologías de estudio - Universidad.

* Magister en Didáctica, Universidad de Buenos Aires. Licenciada y Profesora en Enseñanza Media y Superior en Ciencias de la Educación, Universidad de Buenos Aires. Doctoranda de la Facultad de Filosofía y Letras, Universidad de Buenos Aires. Investigadora becaria del Instituto de Investigaciones en Ciencias de la Educación, Facultad de Filosofía y Letras, Universidad de Buenos Aires y Consejo Nacional de Investigaciones Científicas y Técnicas. 
Revista de la Escuela de Ciencias de la Edducación, año 10, número 9, enero a diciembre de 2014. Páginas 271-286. ISSN 1851-6297. ISSN EN LINEA 2362-3349. LA FORMACIÓN PROFESIONAL EN LA UNIVERSIDAD: UNA PROPUESTA A TRAVÉS DE la evaluación mediante casos autenticos. Maria Florencia Di Matteo Demirdjian.

\begin{abstract}
In this article we present prospects for professor and students of a course of degree in administration from University of Buenos Aires on the assessment based on real cases and we describe perceptions of students on their study methods on this kind of evaluation. The methodology was a case study in a qualitative perspective.

Learning evaluation based on authentic cases constitutes a modality that allows developing skills required for professional training. Demands on an applied use of knowledge and the development of strategies for action on real cases, position the student as a future professional. It happens in an institutional frame in which the predominant teaching practices are whose teaching practices are conceptual theoretical development.

The evaluation is inside teaching practices which includes work in groups, development of oral skills, and self-assessment. The concept of authenticity is related to real character of the cases. The students' study methods are continuous from resolution of tasks in classes and focused on the acquisition of content from its application in the analysis of the cases.
\end{abstract}

\title{
Key Words
}

Learning assessment - Case method - Professional training - Study methods - University.

\section{Introducción}

El estudio de la evaluación de los aprendizajes en la universidad implica situarla en un entrecruzamiento de diversas dimensiones que tomamos como marco contextual para comprender su lugar en la formación profesional.

En primer lugar, durante los últimos años se experimentaron cambios a nivel social y laboral que generaron nuevas demandas a la educación superior, y junto con ella, transformaciones en las funciones y requerimientos de la evaluación. Algunas de esas modificaciones implican el pasaje de la división del trabajo al trabajo en equipo, de la mera ejecución a la participación en la planificación, del trabajo dirigido externamente al auto dirigido, del control externo a la responsabilidad propia. Es decir, opera un tránsito de la calificación para la tarea, a la competencia del trabajador.

Seguidamente, señalamos que la evaluación de los aprendizajes en la universidad presenta un carácter crítico por su responsabilidad en acreditar saberes y competencias profesionales.

Los avances epistemológicos y teóricos producidos en el campo de la pedagogía universitaria y, dentro de ella, en el de la evaluación de los aprendizajes constituyen un tercer elemento contextual que es necesario mencionar. Aquellos han contribuido a la búsqueda de alternativas de evaluación que permitieran manifestar desempeños complejos propios de la actuación profesional y habilidades ligadas al aprender a lo largo de toda la vida. Los mencionados desarrollos instalaron el interés por generar renovadas prácticas de evaluación, 
Revista de la Escuela de Ciencias de la Educación, año 10, número 9, enero a diciembre de 2014. Páginas 271-286. ISSN 1851-6297. ISSN EN LINEA 2362-3349. LA FORMACIÓN PROFESIONAL EN LA UNIVERSIDAD: UNA PROPUESTA A TRAVÉS DE la eValuación mediante casos autenticos. Maria Florencia Di Matteo DemiRdjian.

resignificando su sentido pedagógico, la función de mejoramiento de la tarea de enseñar y del proceso de aprender y ampliando el repertorio de formatos e instrumentos de evaluación.

En el marco de la problemática de la evaluación de los aprendizajes en la universidad y sus vinculaciones con la formación profesional situamos al enfoque de Evaluación Auténtica (Wiggins, 1990). Éste enfatiza el carácter real de las tareas propuestas, incluyendo problemas mal definidos, con límites borrosos y múltiples posibilidades de respuesta. Propone situaciones complejas tomadas del mundo real, o bien cercanas a él, que los estudiantes deben comprender y resolver poniendo en juego saberes previos y estrategias. Al ser tareas ligadas al mundo y a las necesidades reales, son potenciales herramientas para el futuro desempeño profesional; su aprovechamiento para la formación estará dado por el tipo de trabajo demandado.

Entre todos los formatos de evaluación posible para este estudio tomamos el del método de casos, definido como un recurso o herramienta para la enseñanza de un tema. El caso se presenta a través de una narración: un relato en el que se describe una historia, el acontecer de un hecho y las acciones de personajes.

En este artículo caracterizamos la propuesta de formación para la profesión a través del uso de casos auténticos en la evaluación en una cátedra de la Licenciatura en Administración de la Universidad de Buenos Aires. Junto a ello, describimos la perspectiva de la docente y los estudiantes sobre esta modalidad de evaluación y las percepciones de estos últimos sobre sus modalidades de estudio puestas en juego.

Para alcanzar estos objetivos adoptamos un estudio de caso con enfoque metodológico cualitativo. Los materiales empíricos obtenidos y analizados consistieron en documentación (el plan de estudios de la carrera y el programa de la asignatura), observaciones de clases -un total de 14 horas- y de instancias de evaluación a través de un examen - 2 horas- $y$ entrevistas (a la docente del curso y a seis estudiantes seleccionados). Asimismo aplicamos una encuesta destinada a todos los estudiantes del curso ( $n$ : 59).

En el primer apartado contextualizamos la propuesta de evaluación en el marco de un determinado enfoque disciplinar y de las prácticas de enseñanza. Luego, caracterizamos la propuesta de evaluación desde un análisis didáctico analizando allí la perspectiva de los actores del caso. Seguidamente describimos las modalidades de estudio de los alumnos de acuerdo considerando sus propias percepciones. Algunas de las conclusiones indican que la evaluación basada en casos auténticos favorece el desarrollo de habilidades requeridas para la profesión: el uso aplicado de los conocimientos adquiridos, metodologías de análisis, trabajos en grupo, la oralidad y la autoevaluación. 
Revista de la Escuela de Ciencias de la Educación, año 10, número 9, enero a diciembre de 2014. Páginas 271-286. ISSN 1851-6297. ISSN EN LINEA 2362-3349. LA FORMACIÓN PROFESIONAL EN LA UNIVERSIDAD: UNA PROPUESTA A TRAVÉS DE la evaluación mediante casos autenticos. Maria Florencia Di Matteo Demirdjian.

\section{El enfoque disciplinar en la clase, marcos de la evaluación con casos.}

En esta sección presentamos los aspectos que contextualizan a la evaluación basada en el método de casos empleado por la cátedra del estudio. Describimos la propuesta de enseñanza considerando la concepción de la disciplina que allí se expresa y los rasgos de la clase que expresan la alineación entre la enseñanza y la evaluación.

\subsection{La especificidad disciplinar y del enfoque adoptado.}

Observamos una coherencia entre los propósitos formativos enunciados en el plan de estudios y los adoptados por la cátedra. El diseño curricular de la Licenciatura en Administración (1997) señala que promueve la formación de un graduado con conocimientos y capacidades que le permitan analizar e intervenir en organizaciones tomando como modelos los alejados de análisis clásicos. La coherencia con el programa se halla, en tanto se proponen nuevas maneras de enfocar y resolver problemas en las organizaciones del futuro.

Se destaca también una propuesta de enseñanza enmarcada en una cierta forma de pensar la disciplina en cuestión. La cátedra de Planeamiento a Largo Plazo corresponde a una asignatura que toma aportes de distintos marcos teóricos. Entre estos últimos adquiere relevancia el paradigma de la complejidad que presenta una mirada contextual y sistémica y brinda otra forma de entender al mundo de la administración y de los negocios "más allá de lo numérico" tal como señalan estudiantes entrevistados. Este habilitar a nuevas maneras de pensar el planeamiento y las organizaciones es uno de los aspectos que, según los alumnos, constituye un rasgo distintivo de esta cátedra.

El enfoque de la disciplina y de las clases adoptado es descripto en comparación con otras del mismo ámbito institucional en el que se insertan y a las que denominan tradicionales. En la Facultad de Ciencias Económicas, los estudiantes y la docente caracterizan a las clases tradicionales desde un paradigma en el que los temas se presentan desde una sola mirada y sin discusión con otras perspectivas, alejando la posibilidad de promover un pensamiento crítico. Así describen a las clases uno de los estudiantes:

Entrevistadora:- ¿Cuál sería el modelo clásico al que referiste en la encuesta?

Estudiante:- El modelo clásico es clases teóricas donde no tenés participación, tiene que ver con el viejo paradigma que te encierra en sí mismo, en el sentido de que tenés sistemas abiertos y cerrados. La facultad sigue siendo un sistema cerrado, donde se siguen metiendo en sus mismos temas y no se abre a algo interdisciplinario. (...) "En la facultad (...) muchas veces tenés la teoría y después recién la ves cuando salís a la calle o en tu trabajo, pero no 
Revista de la Escuela de Ciencias de la Educación, año 10, número 9, enero a diciembre de 2014. Páginas 271-286. ISSN 1851-6297. ISSN EN LÍNEA 2362-3349. LA FORMACIÓN PROFESIONAL EN LA UNIVERSIDAD: UNA PROPUESTA A TRAVÉS DE la evaluación mediante casos autenticos. Maria Florencia Di Matteo Demirdian.

te muestran casos prácticos en la cursada. (Alberto, Comunicación personal, 17 de mayo de 2010)

De acuerdo con lo expresado por los actores del caso, en las aulas de la Facultad de Ciencias Económicas de la Universidad de Buenos Aires predomina el desarrollo conceptual a través de exposiciones de los docentes sin propuestas de aplicar lo aprendido. Asimismo señalan que en estos espacios no suelen presenciar debates ni intercambios de puntos de vista diferentes. Los estudiantes entrevistados también comentan que, en otras materias, suelen realizar trabajos prácticos que no presentan de manera oral.

\subsection{La clase}

La evaluación a través de casos auténticos no resulta una novedad para los estudiantes del curso de Planeamiento a Largo Plazo. Esto es así en tanto esta metodología de evaluación es también utilizada como estrategia de enseñanza. El uso de casos en las clases adopta las siguientes modalidades: 1) casos reales y artificiales presentados como ejemplos, generalmente durante las exposiciones que realiza el Profesor Titular (1) de la cátedra; 2) casos reales históricos y actuales en distintas escalas organizacionales (supermercados familiares, empresas multinacionales) presentados a través de relatos escritos con descripciones de situaciones y comportamiento de actores en determinadas coyunturas; 3 ) casos artificiales simulados entre el docente y los alumnos a los fines de analizarlos y proponer alternativas de acción y 4) casos reales actuales presentados por sujetos del mundo de los negocios.

En las clases, los contenidos se abordan a través del análisis de casos en donde éste aparece como contenido a aprender y como medio para adquirir otros. En tanto contenido procedimental, el análisis de casos adquiere un tratamiento recurrente ya que se trabaja de esa manera en la mayor parte de los encuentros. Por otra parte, contenidos actitudinales como trabajar en grupo y animarse a exponer oralmente se desarrollan a lo largo de todo el curso.

Esta secuencia supone un tratamiento de los contenidos en profundidad antes que en extensión, característica visualizada tanto por la docente como por los estudiantes. La profundidad se alcanzaría a través del trabajo de análisis que supone, entre otras cosas, la aplicación de los conceptos trabajados en clase y presentes en la bibliografía del curso.

\section{La forma y contenidos de la evaluación y su relación con la enseñanza}

A fin de lograr una mirada amplia de la evaluación, describimos en primer lugar el instrumento y los contenidos implicados en la evaluación destacando las estrechas relaciones entre ellos y la propuesta de enseñanza. Luego, nos referimos a las valoraciones que los propios estudiantes realizan sobre esta 
Revista de la Escuela de Ciencias de la Educación, año 10, número 9, enero a diciembre de 2014. Páginas 271-286. ISSN 1851-6297. ISSN EN LINEA 2362-3349. LA FORMACIÓN PROFESIONAL EN LA UNIVERSIDAD: UNA PROPUESTA A TRAVÉS DE la evaluación mediante casos autenticos. Maria Florencia Di Matteo Demirdjian.

modalidad, explicitando el favorecimiento de aprendizajes comprensivos y ligados a la formación profesional. Por último, presentamos un análisis del lugar que adquieren los criterios de evaluación y su explicitación en los debates acerca de la autonomía y heteronomía en la evaluación ( $y$ en la enseñanza).

\subsection{El formato y los contenidos de evaluación y sus relaciones con la enseñanza}

Un modo de análisis de la evaluación, en lo que hace al instrumento, supone considerarlo desde el punto de vista de su formato. Éste:

(...) alude a la configuración que adopta la evaluación, incluyendo no sólo el tipo de técnica e instrumento empleado sino también las características de la tarea que se propone al estudiante (el desafío cognitivo que supone, su grado de complejidad, su relación con la vida real y la práctica profesional) y el modo de resolución (individual/grupal, en el salón de clases/fuera del salón de clases, entrega inmediata/entrega diferida) (Camilloni y Cols, 2010:10).

Desde un punto de vista técnico, el instrumento de evaluación empleado en el caso corresponde a una prueba escrita con ítems de producción de respuesta. Se realiza de manera individual, en el salón de clases, a libro cerrado y se entrega de manera inmediata (en un tiempo de dos horas reloj).

El parcial contiene un caso real de una conocida fábrica de galletitas e incluye información numérica de investigación de mercado. Como se observa a continuación, las consignas demandan comprensión y aplicación de conceptos, relación e integración de conocimientos y justificación teórica. Los alumnos deben sintetizar e inferir la estructura del planteo más allá de los datos, sacar conclusiones y estrategias o recomendaciones de acción.

1) Relacione los conceptos de Fractales, estructura del Iceberg y Dialéctica Dinámica y aplíquelo al caso.

2) Aplique los siguientes conceptos al caso:
a) Bifurcación
b) Non Locality
c) Paradigma de Simplicidad
d) Valle de la Muerte

3) ¿Cómo clasificaría los modelos mentales y los atractores de cada personaje? ¿Cuál podría ser la visión de Tía Maruca? Justificar

4) ¿Cuáles serían a su criterio los frenos más importantes para el desarrollo del negocio? ¿Cómo aplicaría la ley de la palanca? Si identifica alguna ley más, aplíquela al caso.

5) Dibuje las matrices que sean necesarias y desarrolle la estrategia que sugiere para el futuro de Tía Maruca. (Primer examen parcial, 04 de mayo de 2010)

Si tomamos en cuenta que el tipo de trabajo cognitivo demandado en el examen es semejante al requerido para la resolución de las consignas en las 
Revista de la Escuela de Ciencias de la Educación, año 10, número 9, enero a diciembre de 2014. Páginas 271-286. ISSN 1851-6297. ISSN EN LINEA 2362-3349. LA FORMACIÓN PROFESIONAL EN LA UNIVERSIDAD: UNA PROPUESTA A TRAVÉS DE la eValuación mediante casos autenticos. Maria Florencia Di Matteo Demirdjian.

clases, es posible expresar que las consignas poseen un grado de complejidad adecuado.

La descripción técnica del instrumento de evaluación, coincide con la realizada por los estudiantes, quienes señalan en las entrevistas que este parcial fue práctico o teórico- práctico y, desde las encuestas agregan que consistió en la resolución de una situación problemática/ un caso usando el conocimiento disponible (2).

Los resultados indican que hay un tratamiento del contenido en diferentes facetas o tipos. Así, se hace visible el valor que adquiere la enseñanza de temas en términos de procedimientos, habilidades y actitudes además de los conceptos, leyes y principios (Coll, 1992).

Los contenidos conceptuales requeridos para resolver el examen son coherentes con los enseñados. La prueba demanda aplicar conceptos (visión, fractales, puntos de bifurcación) y principios y leyes (modelos mentales, paradigma de simplicidad y complejidad).

La misma alineación entre la enseñanza y la evaluación encontramos con los contenidos del orden de las habilidades. En el parcial se evalúa el uso de procedimientos de análisis de los casos y la identificación y señalamiento de las acciones que, como futuros profesionales, los estudiantes promoverían en la situación del relato. Otros contenidos de este orden son: la clasificación, la capacidad de realizar inferencias sobre el caso al identificar los conceptos y teorías a aplicar en ese material empírico, de inferir los problemas y de emplear los conceptos. El saber procedimental de análisis de casos no es directamente evaluado, sino a través de las respuestas producidas por los alumnos.

En lo que respecta a los contenidos del orden de las actitudes, se encuentra implícito el valor de los casos para analizar las consignas. Consideramos, además, que la evaluación de este tipo de contenidos resulta dificultosa en instancias de evaluación presenciales y escritas.

Hemos señalado la coherencia entre el tipo de contenido y los desafíos cognitivos de la enseñanza y los de la evaluación destacando así la alineación en la propuesta. Estas estrechas relaciones son también visualizadas por los alumnos del curso quienes señalan, por su parte, la cercanía entre las demandas cognitivas de los trabajos prácticos o actividades realizadas en clase y las requeridas desde el parcial. Algunos señalan: "hubo continuidad y similitud entre ambos", "el parcial no fue una sorpresa, fue un reflejo de las clases y parecido a los debates dados en clases" (Alexia, Augusto, Comunicaciones personales, 19 de mayo de 2010)

Por otra parte, la mayoría de los alumnos encuestados expresa que antes del parcial conocía cuál sería la modalidad; principalmente lo imaginaban por el tipo de tareas realizadas en clase y también señalan que lo sabían por las anticipaciones realizadas por la docente; en menor medida disponían de esta 
Revista de la Escuela de Ciencias de la Educación, año 10, número 9, enero a diciembre de 2014. Páginas 271-286. ISSN 1851-6297. ISSN EN LINEA 2362-3349. LA FORMACIÓN PROFESIONAL EN LA UNIVERSIDAD: UNA PROPUESTA A TRAVÉS DE la evaluación mediante casos autenticos. Maria Florencia Di Matteo Demirdian.

información a partir de comentarios de compañeros que cursaron en años anteriores.

Estas declaraciones podrían analizarse considerando el concepto de "trabajo familiar" (Doyle, 1986) que indica que la tarea es conocida de antemano por el estudiante. El disponer de mecanismos cognitivos internalizados (desarrollados a través de las actividades de clase) facilita la resolución de la prueba.

\subsection{El método de casos en la evaluación (y en la enseñanza) desde el punto de vista de los estudiantes: aportes para la comprensión y la formación profesional}

La modalidad de evaluación basada en casos es considerada por los estudiantes encuestados como útil/productiva; interesante/innovadora y ni fácil ni difícil/normal (3).

Gráfico 1. Consideración de la evaluación por parte de los estudiantes

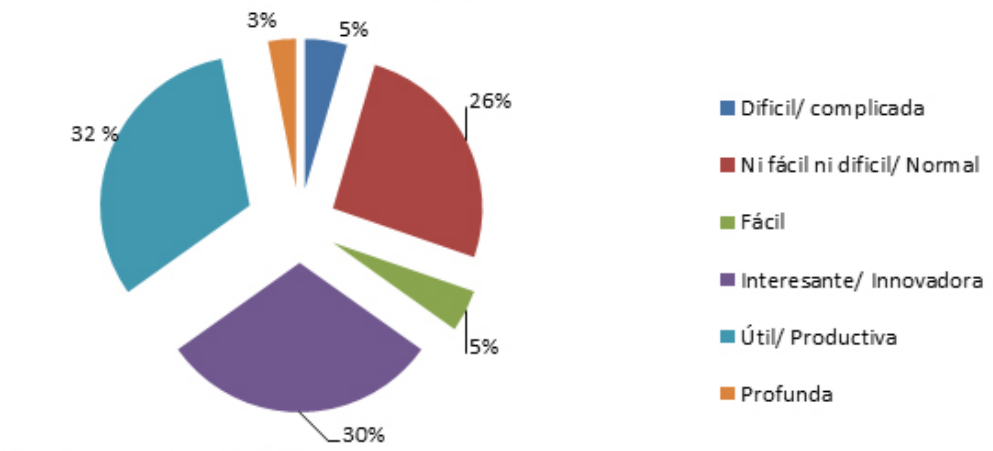

Fuente: encuesta propia. N: 59

La utilidad / productividad de la evaluación haría referencia a su relación con el aprendizaje comprensivo y con la posibilidad de transferir lo aprendido a la práctica profesional. El escaso uso de metodologías de este tipo en la Facultad de Ciencias Económicas permitiría explicar su consideración como interesante / innovadora. Según señalan los estudiantes entrevistados, predominan prácticas de enseñanza y evaluación basadas en desarrollos conceptuales sin aplicación a situaciones prácticas. La apreciación de la evaluación como ni fácil ni difícil / normal remitiría a la coherencia entre las prácticas de enseñanza y las de evaluación; si el trabajo con casos es frecuente en las clases, la evaluación no tendría por qué resultar difícil, extraña o fuera de lo normal.

Este análisis permite expresar que el instrumento de evaluación presentaría una validez manifiesta, es decir una significatividad percibida por los estudiantes como instancia adecuada para la evaluación (Camilloni, 1998). 
Revista de la Escuela de Ciencias de la Educación, año 10, número 9, enero a diciembre de 2014. Páginas 271-286. ISSN 1851-6297. ISSN EN LINEA 2362-3349. LA FORMACIÓN PROFESIONAL EN LA UNIVERSIDAD: UNA PROPUESTA A TRAVÉS DE la eValuación mediante casos autenticos. Maria Florencia Di Matteo DemiRdjian.

La preferencia por esta modalidad de evaluación es otros de los aspectos indagados en la encuesta empleada a estudiantes. Aquí, casi el $60 \%$ se inclina favorablemente a ella en tanto que un $43 \%$ señala no tener preferencias. Son causas de las preferencias: el favorecimiento de la comprensión, aplicación y reflexión sobre los conceptos, su cercanía y ubicación en el ámbito profesional y su promoción de la articulación y aplicación de la teoría a la práctica. Estas respuestas otorgan valor a los aportes de la evaluación para la práctica y la profesión. Otros estudiantes indican que este tipo de evaluación permite demostrar lo aprendido y favorece un pensamiento práctico y crítico.

Como punto favorable, es que podés aplicar toda la gama de conceptos teóricos en un caso práctico que es muy bueno tenerlo en mente. Los conceptos, en mi caso, yo no los aprendo por repetir una y otra vez y no creo que la gente tampoco pueda. El concepto se aprende cuando uno los internaliza y cuando los plasma en un ejemplo. Toda la carrera trato de aplicarla a mi trabajo, ahí es cuando uno aprende. En este examen te haya ido bien o mal, te sirve para internalizar los conceptos más allá de la nota refleje una cosa u otra. (Alanestudiante-, Comunicación personal, 1 de junio de 2010)

Para algunos alumnos y la docente del curso esta modalidad de evaluación permite "bajar la presión de tener que estudiar para el parcial" (Victoriaprofesora-, Comunicación personal, 3 de marzo de 2011) y no genera estrés a los estudiantes por ser conocida de antemano y practicada en las clases. Ello podría relacionarse con los resultados de investigaciones que correlacionan niveles de ansiedad con rendimiento académico. Las conclusiones de estos estudios indican que la ansiedad se liga a pensamientos que interfieren en el aprendizaje y se correlacionan negativamente con bajos niveles de rendimiento en exámenes escritos (Chiecher, Donolo y Rinaudo, 2003). Si bien no contamos con las calificaciones de cada estudiante, sabemos por la observación de la última clase que una amplia mayoría de los estudiantes aprobó la materia.

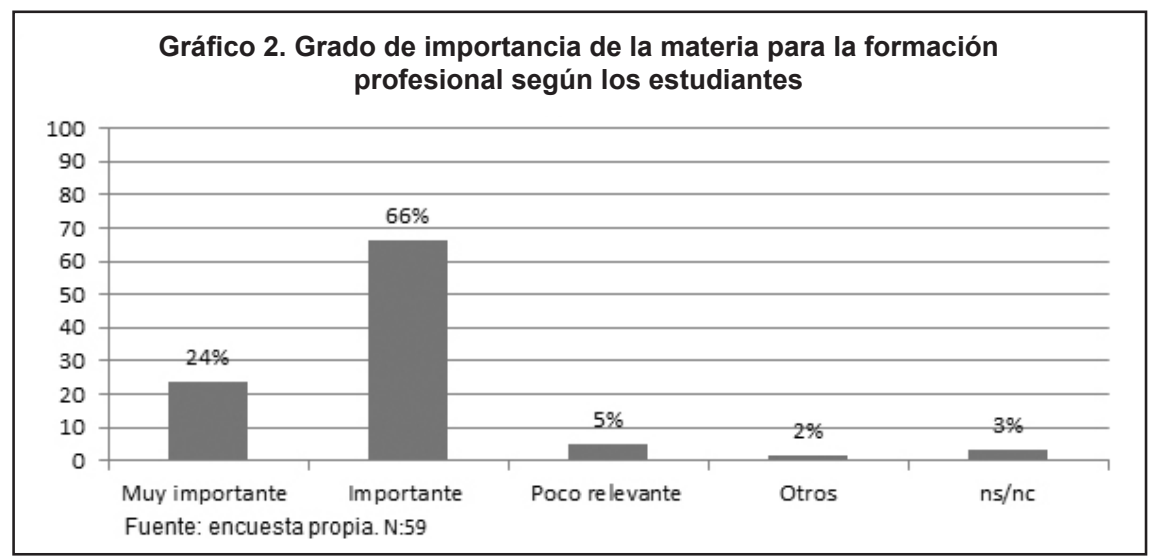


Revista de la Escuela de Ciencias de la Educación, año 10, número 9, enero a diciembre de 2014. Páginas 271-286. ISSN 1851-6297. ISSN EN LINEA 2362-3349. LA FORMACIÓN PROFESIONAL EN LA UNIVERSIDAD: UNA PROPUESTA A TRAVÉS DE la evaluación mediante casos autenticos. Maria Florencia Di Matteo Demirdjian.

Por parte de los estudiantes no hay referencia a dificultades y desventajas en este tipo de evaluación. Algunos remiten a la falta de habituación a esta modalidad, que perciben como tal solamente en las primeras clases.

Así como hemos observado, los aportes del método de casos (en la enseñanza y en la evaluación) a la formación profesional son uno de los aspectos más salientes en este estudio. De acuerdo con lo expresado en el siguiente gráfico la mayoría de los estudiantes considera que la materia es entre importante y muy importante para esta formación.

Argumentos (4) sobre esta importancia refieren a que los contenidos y metodologías de trabajo brindan aportes a su formación. Esos saberes construidos son concebidos por los estudiantes como "herramientas para ampliar las formas de pensar" que contribuyen a una "formación integral" (Alexia, Alan -estudiantes-, Comunicaciones personales, 19 de mayo de 2010 y 1 de junio de 2010).

Estas impresiones permitirían reflexionar sobre la importancia que adquiere esta materia tanto para la formación centrada en el mundo del trabajo como para aquella focalizada en el análisis y pensamiento crítico al que la universidad se espera que contribuya (Menin, 2001).

Esta propuesta de enseñanza y de evaluación también constituiría un acercamiento a la formación profesional en tanto son tareas de la profesión: analizar situaciones de mercado a partir del uso y aplicación de diversas herramientas y replantear estrategias empresarias. Otra contribución al desarrollo profesional se daría en el desarrollo de la oralidad en las clases, una habilidad necesaria para el desempeño laboral.

\subsection{Los criterios de evaluación.}

Entre las principales demandas de los estudiantes encontramos el pedido para que los docentes realicen devoluciones sobre los trabajos prácticos realizados. Según indican, estas retroalimentaciones permitirían resolver dudas, conocer aciertos y logros. Así lo señalan:

(...) a mí me gustaría (...) que te hagan una devolución de los trabajos que vos presentás porque no necesariamente uno capta todo. Estaría bueno que los profesores, al margen de recibir y dártelo en clase, que no se habla todo, te hagan una devolución de los trabajos. (Alberto-estudiante-, Comunicación personal, 17 de mayo de 2010).

La docente señala que ese trabajo se realiza en clase y que las correcciones sobre el trabajo del grupo que expone su análisis del caso en cuestión, debieran servir para que el resto autoevalúe sus propias producciones. Así lo señala la profesora: "Por ahí el obstáculo es: "pero ¿ustedes no nos van a dar una respuesta por escrito?" Las correcciones se las van a llevar ustedes desde la clase. Lo que hacemos es que ellos entiendan y que ellos marquen 
Revista de la Escuela de Ciencias de la Educación, año 10, número 9, enero a diciembre de 2014. Páginas 271-286. ISSN 1851-6297. ISSN EN LINEA 2362-3349. LA FORMACIÓN PROFESIONAL EN LA UNIVERSIDAD: UNA PROPUESTA A TRAVÉS DE la eValuación mediante casos autenticos. Maria Florencia Di Matteo Demirdian.

sus correcciones." (Victoria-profesora-, Comunicación personal, 3 de marzo de 2011). Estos comentarios nos interrogan ¿hay que ofrecer retroalimentación individual o grupal? (Anijovich, 2010).

La posibilidad de explicitar y trabajar sobre los criterios habilitaría a que el estudiante tome una postura más activa en su propio proceso de evaluación, propiciando que esa evaluación externa se convierta en autoevaluación y de manera interactiva favorezca y contribuya al desarrollo de la metacognición. Señalamos aquí la importancia que adquiere la autoevaluación en tanto competencia necesaria para un buen ejercicio profesional.

\section{Modalidades de estudio para la evaluación basada en casos auténticos: el punto de vista de los estudiantes}

Entre las actividades de preparación para la evaluación, los estudiantes señalan las que se presentan a continuación y en el siguiente orden de importancia (5): 1) leer los materiales y resolver guías / cuestionarios u otras actividades dadas por el profesor; 2) repasar los apuntes de clase y los resúmenes de los textos; 3 ) revisar las actividades realizadas en clase y los apuntes a fin de identificar lo que el profesor considera importante y 4) tratar de relacionar lo visto en clase y las explicaciones del profesor con las ideas planteadas en los textos.

Observamos, por un lado, el valor otorgado por los estudiantes a los materiales de lectura como fuente de información válida para el estudio; por otro, la valoración de lo que sucede en las clases como indicador de lo considerado importante para el estudio.

En cuanto a los tiempos de preparación para el examen, todos los estudiantes destacan una modalidad continua que no diferencia entre instancias de estudio durante las clases y destinadas específicamente para el parcial.

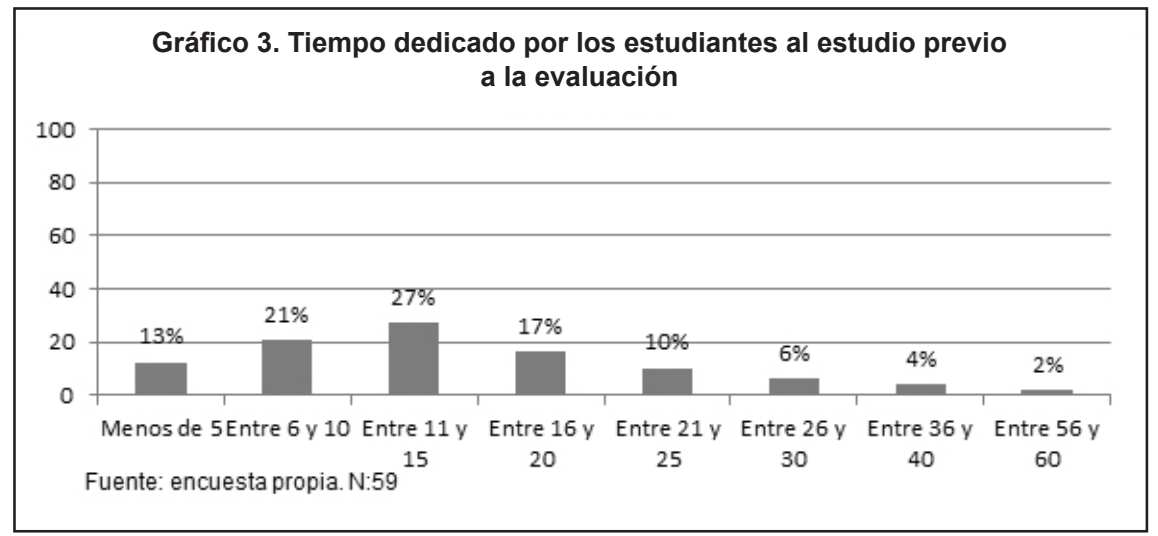


Revista de la Escuela de Ciencias de la Educación, año 10, número 9, enero a diciembre de 2014. Páginas 271-286. ISSN 1851-6297. ISSN EN LINEA 2362-3349. LA FORMACIÓN PROFESIONAL EN LA UNIVERSIDAD: UNA PROPUESTA A TRAVÉS DE la evaluación mediante casos autenticos. Maria Florencia Di Matteo Demirdian.

En el mismo cuestionario, los estudiantes aportan respuestas por fuera del tiempo horario: afirman que el estudio es realizado durante toda la cursada. "Desde que empezó el cuatrimestre, lectura promedio diaria una hora" (Alanestudiante-, Comunicación personal, 1 de junio de 2010). Analizar casos y resolver preguntas a modo de problemas es una tarea frecuente en las clases que los ayuda a apropiarse progresivamente de los conceptos y de las formas de análisis. "Se fue preparando desde la realización de trabajos prácticos similares, por eso no fue 'empezar a estudiar de cero" (Sofía-estudiante-, Comunicación personal, 15 de junio de 2010).

En relación con la modalidad de estudio con otros, casi la mitad de los encuestados señalan estudiar solos. Este punto llama la atención ya que los estudiantes, por un lado, comentan que el estudio es a diario a partir de la realización de análisis de casos de manera grupal en cada clase. Por otra parte, ante la pregunta ¿Estudia solo/a o en grupo?, entre las opciones dadas, seleccionan aquella que refiere a lo individual.

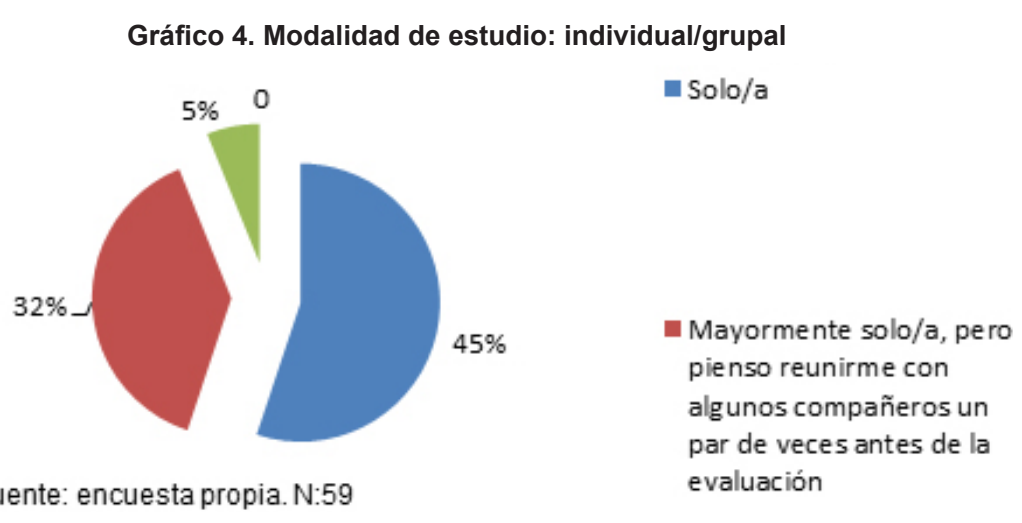

Una mirada diferente sobre el trabajo grupal es realizada por los estudiantes durante las entrevistas. Allí señalan que la tarea en equipo favorece un análisis recursivo de los casos: de manera individual, con el pequeño grupo y con la clase.

Entrevistadora:- ¿Cómo resuelven los trabajos prácticos?

Estudiante:- La metodología nuestra es cada uno lleva leído el caso particular, la teoría es lo de clase, algunos sí otros no, nos juntamos y ahí empezamos "yo creo que esto, yo creo lo otro", vas tomando lo de cada uno (...) está bueno, vos tenés una análisis previo con tus compañeros del grupo y después, en definitiva vas viendo tres veces las cosas como para ver las interpretaciones.

Entrevistadora:- ¿Cuáles son esas tres veces? 
Revista de la Escuela de Ciencias de la Educación, año 10, número 9, enero a diciembre de 2014. Páginas 271-286. ISSN 1851-6297. ISSN EN LINEA 2362-3349. LA FORMACIÓN PROFESIONAL EN LA UNIVERSIDAD: UNA PROPUESTA A TRAVÉS DE la eValuación mediante casos autenticos. Maria Florencia Di Matteo DemiRdjian.

Estudiantes:- Solo, con mis compañeros y otra vez en clase. (Albertoestudiante-, Comunicación personal, 17 de mayo de 2010)

La valoración del trabajo con otros es realizada también a partir de los debates dados en las clases. Para los estudiantes el trabajo de análisis de casos elaborado de manera grupal y la puesta en común realizada de manera oral y clase a clase favorece un estudio continuo y, por esta vía, el aprendizaje.

En este proceso de intercambios grupales se intercambian pareceres, se expresan acuerdos y desacuerdos que también se exponen como objeto de análisis. Señalamos en este punto el lugar constructivo que promueve el trabajo colaborativo en el aprendizaje y en la construcción de saberes (Perret- Clermont y Nicolet, 1988; Salomon, 1993).

De acuerdo con lo señalado, podemos también considerar que el trabajo grupal, si es bueno, puede ayudar al aprendizaje de los contenidos, los relacionados con la tarea específica desarrollada por el grupo y los saberes relativos al trabajo grupal (Camilloni, 2010) a través del intercambio desde lo cognitivo y por el sostén emocional y motivacional.

\section{Conclusiones}

Uno de los aspectos más salientes del estudio que da marco a los resultados obtenidos es la coherencia observada entre la propuesta de enseñanza y la de evaluación. Esta alineación refiere tanto a los contenidos enseñados y evaluados como a las demandas cognitivas implicadas en ambos procesos.

La evaluación a través de casos auténticos requiere del estudiante la puesta en juego de procesos cognitivos de orden intermedio y superior requeridos para, por ejemplo, resolver una situación problemática / un caso usando el conocimiento disponible, ello implica relacionar información, conceptos e ideas, sacar conclusiones, elaborar inferencias a partir de la información provista en los relatos de los casos. Se trata de desafíos cognitivos conocidos que implican acciones mentales promovidas desde las actividades de clase. La práctica del análisis de casos en las clases baja la ansiedad previa de los estudiantes a los exámenes y los prepara para su resolución exitosa. En este punto nos preguntamos si la evaluación reproduce lo realizado en clases podría atentar contra el sentido de autenticidad de las tareas propuestas y la demanda cognitiva de comprensión.

Una adecuada resolución de la prueba basada en el análisis de casos implica el manejo y aplicación de contenidos de orden conceptual y el propio manejo del análisis de los casos; se trata de un saber hacer en relación con los datos que requiere práctica y ella implica un trabajo sobre los contenidos en profundidad, lo cual implica dejar de lado ciertos contenidos para abocarse a otros. Esto podría considerarse un punto crítico de la metodología de evaluación (y de enseñanza) basada en casos, aspectos que no aparecen señalados por los estudiantes ni por la docente. 
Revista de la Escuela de Ciencias de la Educación, año 10, número 9, enero a diciembre de 2014. Páginas 271-286. ISSN 1851-6297. ISSN EN LINEA 2362-3349. LA FORMACIÓN PROFESIONAL EN LA UNIVERSIDAD: UNA PROPUESTA A TRAVÉS DE la evaluación mediante casos autenticos. Maria Florencia Di Matteo Demirdjian.

En lo que respecta a la autenticidad de las prácticas de evaluación señalamos que si bien los casos empleados refieren a situaciones y personajes reales, su ubicación en un marco de enseñanza cuestiona esta autenticidad. Entre los aspectos que contribuyen a la artificiosidad (Souto, 2010) encontramos que la resolución de las tareas de evaluación se encuentran acotadas por su ubicación en el marco de una institución del sistema educativo y en el espacio y tiempo del aula; es decir, los estudiantes resuelven los parciales en la situación artificial de la clases y por fuera del ámbito de trabajo real.

No obstante lo señalado, la propuesta de evaluación basada en casos auténticos se convierte una instancia de formación para la profesión. Esta modalidad de trabajo posiciona al estudiante en un lugar diferente en su vinculación con el conocimiento a través de un uso activo en vistas a analizar casos y a proponer estrategias o recomendaciones de acción, funciones que deberán desarrollar en su futura vida laboral. Se trata de saberes requeridos para el ejercicio de la profesión, aquellos que certifica la evaluación en la universidad.

La contribución a la formación laboral queda también expresada en tanto esta modalidad de evaluación en la cátedra estudiada fomenta el trabajo en equipo, propio del trabajo profesional actual, la oralidad, también necesaria para un adecuado desempeño laboral y la autoevaluación, competencia necesaria para la reflexión sobre la propia tarea y el rol.

En relación con esto último, señalamos especialmente el lugar que ocupan el trabajo sobre los criterios de evaluación en Planeamiento a Largo Plazo. Tal como señalamos, observamos tensiones entre las demandas de los estudiantes y la postura de la docente. Mientras los primeros reclaman devoluciones individualizadas, desde la cátedra se enfatiza que las retroalimentaciones se realicen de manera grupal a fin de que los estudiantes adquieran habilidades para autoevaluarse. Estas tensiones remiten a una de las discusiones actuales en el campo de la evaluación: el desarrollo de la autonomía del sujeto que aprende a partir de un trabajo de autoevaluación. Pero la autoevaluación no se desarrolla espontáneamente, el docente debe considerarla como un contenido de enseñanza.

Finalmente, para analizar la relación entre modalidades de estudios y prácticas de evaluación, tomamos el concepto de validez de retroacción (Camilloni, 1998). Este designa el fenómeno por el cual las tareas de aprendizaje y las modalidades de estudio se configurarían según criterios y requisitos inferidos por los alumnos una vez conocidas las modalidades de evaluación empleadas en una determinada asignatura. Por un lado, encontramos que los estudiantes estudian tomando en cuenta las demandas y actividades de las clases e infiriendo aquello que creen es considerado importante por el profesor; y por otra parte, señalamos una coherencia entre la enseñanza y la evaluación. Por lo expuesto, creemos que, si los alumnos estudian considerando los requerimientos de las clases y del profesor, estarían adoptando una modalidad de 
Revista de la Escuela de Ciencias de la Educación, año 10, número 9, enero a diciembre de 2014. Páginas 271-286. ISSN 1851-6297. ISSN EN LINEA 2362-3349. LA FORMACIÓN PROFESIONAL EN LA UNIVERSIDAD: UNA PROPUESTA A TRAVÉS DE la evaluación mediante casos autenticos. Maria Florencia Di Matteo Demirdian.

estudio que tendría en cuenta los requerimientos de la evaluación. Entonces, estos resultados expresarían que los estudiantes adoptan una modalidad de estudio y un enfoque de aprendizaje cercanos a las demandas cognitivas requeridas desde los formatos de evaluación. Estos resultados permiten pensar en la importancia del rol docente en tanto facilitador de procesos reflexivos sobre las operaciones cognitivas implicadas en las tareas de aprendizaje y en las de evaluación.

\section{Notas Bibliográficas}

* Agradezco especialmente a la Profesora Alicia Camilloni, directora de mi tesis de Maestría en Didáctica, estudio del que se desprende este artículo. A la Doctora Diana Mazza, agradezco la lectura atenta y los valiosos comentarios sobre este trabajo.

(1) El Profesor Titular es quien está a cargo de la cátedra, diseña el programa y coordina el trabajo.

(2) Esta opción es elegida por los estudiantes entre un conjunto de respuestas dadas en la encuesta empleada.

(3) Estas categorías corresponden a respuestas dadas a partir de opciones cerradas a la pregunta sobre cómo les resultó la evaluación en esta materia según su estilo de estudio. Vale aclarar que los estudiantes podían elegir más de una opción posible.

(4) Estas respuestas surgen a partir del análisis de las repuestas a la siguiente pregunta abierta del cuestionario: ¿Qué impresión general tiene acerca del nivel de exigencia de esta materia?

(5) El cuestionario presenta la pregunta “¿Qué actividades cree que debió realizar para prepararse para la evaluación?" y veinte opciones dadas sobre diferentes actividades de preparación para el parcial. Entre ellas los estudiantes debían elegir las cinco consideradas más importantes.

\section{Referencias Bibliográficas}

- $\quad$ Anijovich, R. (2010). La evaluación significativa. Buenos Aires: Paidós.

- Camilloni, A. (1998). La calidad de los programas de evaluación y de los instrumentos que los integran en Camilloni et al: La evaluación de los aprendizajes en el debate didáctico contemporáneo (67-92). Buenos Aires: Paidós.

- - (2010). "La evaluación de trabajos elaborados en grupo" en Anijovich, R. (comp.) La evaluación significativa. Buenos Aires: Paidos.

- Camilloni, A. Cols, E. (2010). La problemática de la investigación didáctica: El caso de una investigación sobre formatos de evaluación de los aprendizajes y sus relaciones con las modalidades de estudio de los alumnos universitarios En Wainerman, C. y Di Virginia, M. (comps) El quehacer de la investigación en educación (pp. 127-141). Buenos Aires: Manantial.

- Chiecher, A., Donolo, D., Rinaudo, M. C. (2003). Motivación y uso de estrategias en estudiantes universitarios: Su evaluación a partir del Motivated Strategies Learning Questionnaire. Anales de Psicología (pp 107-120), ISSN 0212-9728, 19 (1).

- Coll, C. et al (1992). Los contenidos en la Reforma. Enseñanza y aprendizaje de conceptos, procedimientos y actitudes. Buenos Aires: Santillana. 
Revista de la Escuela de Ciencias de la Edducación, año 10, número 9, enero a diciembre de 2014. Páginas 271-286. ISSN 1851-6297. ISSN EN LINEA 2362-3349. LA FORMACIÓN PROFESIONAL EN LA UNIVERSIDAD: UNA PROPUESTA A TRAVÉS DE la evaluación mediante casos autenticos. Maria Florencia Di Matteo Demirdjian.

- Doyle, W. (1986). Trabajo Académico. Traducción de la cátedra de Didáctica II, Facultad de Filosofía y Letras, UBA del artículo aparecido en T. M. Tomilson \& H. J. Walberg, Academic work and educational excellence: Raising student productivity. Berkeley: McCutchan.

- Menin, O. (2001 [2006]). Pedagogía y Universidad. Curriculum, didáctica y evaluación. Rosario: Homo Sapiens Ediciones.

- $\quad$ Perret Clermont, A. y Nicolet, M. (1988) Interactuar y conocer. Desafíos y regulaciones sociales en el desarrollo cognitivo. Buenos Aires: Miño y Dávila.

- Salomon, G. (1993). Cogniciones distribuidas. Consideraciones psicológicas y educativas. Buenos Aires: Amorrortu.

- Souto, M. (2010). El carácter de "artificio" del dispositivo pedagógico en la formación para el trabajo. Buenos Aires: OPFyL.

- Wiggins, G. (1990). The case for authentic assessment. Practical Assessment, Research \& Evaluation, 2 (2).

\section{Documentos:}

- Plan de estudios Licenciatura en Administración, Universidad de Buenos Aires

- Programa de Planeamiento a Largo Plazo, Facultad de Ciencias Económicas, Universidad de Buenos Aires

- $\quad$ Primer examen parcial 04/05/2010

- Entrevistas personales a docente (03/03/2011) y a estudiantes (17/05/2010; 01/06/2010; $15 / 06 / 2010$ y $16 / 06 / 2010)$. 